\title{
Die mehrsprachige Universität und ihre Stereotype \\ Einblicke in das Forschungsprojekt VAMUS
}

\author{
Marion Flach (Innsbruck)
}

\begin{abstract}
Science and academic education are determined by discussion and scientific exchange across borders of both languages and countries. Consequently, universities can be understood as melting-pots: places where, traditionally, different forms of multilingualism are practiced. Academic staff, students, and administrative staff have different perceptions of multilingualism. Furthermore, they develop their specific and individual attitudes which are presumably influenced by traditions of the faculty or even the discipline. In this paper, I will present first findings of the research project "Interwoven Analysis of Forms of Multilingualism Illustrated by the Example of the University of Salzburg" (OeNB Project $\mathrm{Nr}^{\circ} 15$ 827) which aims to investigate the complex situation described above. In addition, I will focus on perceptions of multilingualism (also including different varieties of the German language). To determine how stereotypes and attitudes towards multilingualism are expressed, there will be a content analysis of interviews. It is shown that there is a difference in the use of attitudes and stereotypes. They differ particularly in matters of social validity. This suggests that it is important as well to have a further look on the theoretical distinction.
\end{abstract}

\section{$1 \quad$ Einleitung}

„Wissenschaft ist auf Diskussion und Austausch über Landes- und Sprachgrenzen hinweg angewiesen und braucht dafür geeignete Verständigungsmittel“" (Dannerer 2008: 59). Dieses Zitat verdeutlicht, dass Wissenschaft - und somit auch universitäre (Aus-)Bildung - auf Mehrsprachigkeit zurückgreifen muss. Nicht zuletzt auf Grund dieses Selbstverständnisses gilt der Interaktionsraum Universität als Bildungs- und Forschungsinstitution seit dem Sprachenwechsel vom Lateinischen zu den Vernakularsprachen (cf. Schiewe 1996: 36-38, 120131) traditionell als mehrsprachig. Es ist jedoch davon auszugehen, dass diverse Aktanten diese mehrsprachige Bildungs- und Diskurssituation sehr unterschiedlich wahrnehmen und für sich selbst nutzen. Daraus können verschiedenste Einstellungen zur Mehrsprachigkeit, vielleicht sogar Stereotype entstehen.

Oftmals ist das (sprachliche) Handeln im mehrsprachigen Umfeld Universität unproblematisch. Die aus den zuvor genannten Wahrnehmungen und Erfahrungen entstehenden kollektiven und individuellen Vorstellungen in Bezug auf die an der Universität vorherrschende sprachliche Vielfalt können aber durchaus zur Akzeptanz oder Ablehnung verschiedener 
(sprachpolitischer) Maßnahmen und Vorgehensweisen führen und damit Einfluss auf das (kommunikative) Miteinander haben.

In diesem Zusammenhang ist zu fragen, was als individuelle, was als kollektive Wahrnehmung eingestuft werden kann und wie dies auf der sprachlichen Ebene festzumachen ist. Wie unterscheiden sich Einstellungen und Stereotype? Können ihnen in weiterer Folge kommunikative Funktionen zugesprochen werden?

Im vorliegenden Beitrag ${ }^{1}$ wird in einem ersten Schritt (Abschnitt 2) die theoretische Basis, auf die sich dieser Artikel stützt, dargelegt. Es wird vor allem darum gehen, den Mehrsprachigkeitsbegriff, von dem hier ausgegangen wird, zu erläutern sowie das zugrunde gelegte Verständnis bzgl. Stereotyp und Attitüde festzuhalten. Attitüden werden in der Forschung häufig „als ein Komplex von Einstellungen, Dispositionen zu Verhalten und tatsächlichem Verhalten verstanden“ (Kaufmann 1997: 196). Grundsätzlich folgt der vorliegende Beitrag dieser Ansicht, da Attitüden immer mehrere Komponenten einschließen (Kognition, Affekte, Verhalten) (cf. Garrett 2010: 23) und immer nur das Konglomerat dieser Komponenten in Form von Attitüden beobachtet und untersucht werden kann. Auf Einstellungen und auch Vorstellungen kann also nicht direkt zugegriffen werden. Die Begriffe Vorstellungen und Einstellungen werden folglich nicht gesondert thematisiert, sondern als Teil der Attitüden gesehen und hier - der Einfachheit halber - synonym verwendet.

An die theoretischen Überlegungen anknüpfend (Abschnitt 3) wird das Datenmaterial vorgestellt, das im Rahmen des Projektes Verknüpfte Analyse von Mehrsprachigkeiten am Beispiel der Universität Salzburg (VAMUS, ÖNB Nr. 15 827) erhoben wird. Dieses Projekt setzt sich mit Formen und Funktionen von Mehrsprachigkeit im universitären Kontext auseinander. In diesem Zusammenhang soll zudem kurz erläutert werden, welche Ziele im angesprochenen Forschungsvorhaben verfolgt werden. Im Anschluss daran werden Methoden und Fragestellungen der vorliegenden Analyse erläutert (Abschnitt 4). In Abschnitt 5 werden drei unterschiedliche Fallbeispiele einer Inhaltsanalyse unterzogen. Abschließend (Abschnitt 6) wird ein Fazit gezogen und versucht, Desiderata in Bezug auf den Umgang mit Stereotypen und Attitüden anzudeuten.

\section{Theoretische Basis}

Damit klar wird, worauf in der Analyse von Mehrsprachigkeit jeweils Bezug genommen wird, ist zunächst zu definieren, was hier unter Mehrsprachigkeit verstanden wird. In einem weiteren Schritt wird dargelegt, wie der Terminus Stereotyp aufgefasst wird und inwieweit er auf einer theoretischen Basis zum Begriff der Attitüde abgegrenzt werden kann.

\subsection{Mehrsprachigkeit}

Die Beschäftigung mit der Heterogenität von Sprache und unterschiedlichen sprachlichen Praktiken hat in der Soziolinguistik bereits eine lange Tradition (cf. Auer 2007: 1). Einen

\footnotetext{
${ }^{1}$ An dieser Stelle möchte ich Jörg Kilian und Erla Hallsteinsdóttir sowie den ReviewerInnen für die konstruktive Kritik an diesem Beitrag sowie für die Literaturhinweise danken, die bei der Überarbeitung eine große Hilfe waren und zur Präzisierung einiger Überlegungen geführt haben.
} 
Überblick über verschiedene Konzepte und Kategorien, die im Laufe der Zeit entwickelt, beforscht und diskutiert wurden, gibt beispielsweise Busch (2013).

Einen sehr offenen Mehrsprachigkeitsbegriff vertritt Franceschini (2011), die folgendermaßen definiert:

Multilingualism conveys the ability of societies, institutions, groups, and individuals to have regular use of more than one language in their everyday lives over space and time. Language is impartially understood as a variety that a group admits to using as a habitual communication code (regional languages and dialects are also included, such as sign languages).

In observing multilingual practices, it is possible to distinguish societal, institutional, discursive, and individual multilingualism.

(Franceschini 2011: 346)

In weiterer Folge sieht Franceschini (2011: 346) Mehrsprachigkeit auf der Basis der menschlichen Fähigkeit, in mehreren Sprachen zu kommunizieren, und versteht dies als Ausdruck hoher kultureller Sensibilität. Ein zentraler Aspekt ist hier ebenfalls, dass Mehrsprachigkeit nicht auf das Abrufen unterschiedlicher Einzelsprachen beschränkt ist, sondern auch verschiedene Varietäten einer Sprache einbindet.

Auch Wandruszka (1979) vertritt einen Mehrsprachigkeitsbegriff, der nicht auf die Abgrenzung von Einzelsprachen abzielt, sondern vielmehr die Vielschichtigkeit der Sprachen mitberücksichtigt. So prägte Wandruzska (1979) die Termini der ,inneren“ und ,äußeren“ Mehrsprachigkeit. Wenn von ,innerer“ Mehrsprachigkeit gesprochen wird, so ist das Varietätenspektrum einer Sprache gemeint, während sich ,äußere“ Mehrsprachigkeit auf unterschiedliche Einzelsprachen bezieht.

Dannerer/Mauser (2013: 8) halten in diesem Zusammenhang fest, ,dass darüber hinaus [über die von Franceschini angenommenen unterschiedlichen Formen von Mehrsprachigkeit hinaus] auch die diskursive Etablierung der Formen der Mehrsprachigkeit zu untersuchen ist.“ Dies kann in weiterer Folge als Ausgangspunkt dafür genommen werden, innere und äußere Mehrsprachigkeit gemeinsam zu betrachten.

\subsection{Stereotype und Attitüden}

In diesem Beitrag wird von einem sozialpsychologisch geprägten Stereotypen-Begriff ausgegangen, bei dem es sich nach Quasthoff (1973: 13) um ein gesellschaftliches Phänomen ${ }^{2}$ handelt, das sich sprachlich manifestiert und ,in sehr enger Beziehung zur sprachlichen Äußerung steht“" (Quasthoff 1973: 13):

The word 'stereotype' is used in at least two different ways: as a technical term which has its origin in social psychology and refers to a certain kind of prejudice, and in everyday language, where it means something like 'constantly repeated', 'meaningless' with respect to verbal cli-

\footnotetext{
${ }^{2}$ Neben dieser sozialpsychologischen Auffassung des Stereotyps gibt es auch einen semantiktheoretisch geprägten Begriff des Stereotyps (cf. Pümpel-Mader 2010), der für diesen Beitrag keine Rolle spielen wird. Ich beziehe mich also nicht auf Konzepte wie jenes von Putnam (1975), das erkenntnistheoretisch geprägt ist oder jenes von Schaff, das auf einer marxistischen Grundlage entwickelt wurde (cf. Klein 1998: 30-38; Konerding 2001: 156160).
} 
chés. In view of this situation, linguistics has to decide, which of these established meaning components it will take up in coining a technical term 'stereotype'.

(Quasthoff 1978: 3)

Nach Allport (1971: 200) ist für die Kategorie des Stereotyps anzunehmen, dass es sich um eine „feste Vorstellung“ handelt, die mit positiven oder negativen Einstellungen zusammenhängt, was Konerding (2001) noch weiter spezifiziert. Er spricht in Bezug auf das Stereotyp von „kognitive[n] Schematisierungen“ (Konerding 2001: 152). Somit beschreibt er es als Reduktion der potentiellen Komplexität der Wahrnehmung der Umwelt „auf die sozial relevanten und sanktionierten Aspekte der Wirklichkeit“". So wirken Stereotype

identitätsstiftend, da sie über die Konsensualität die Erfahrung der Zugehörigkeit zu einer bestimmten Gruppe vermitteln, besonders sichtbar im Kontrast zu sozialen Gruppen mit alternativen Stereotypen

(Lippmann 1922: 96)

Stereotype sind daher, wie Konerding (2001: 152, Hervorhebung MF) ausführt, ,ganz generell als sozial verbindliche, konsensuelle Wahrnehmungs- und Evaluationsschemata zu begreifen“. Als weiteres Merkmal von Stereotypen führt Ladegaard (2011: 87) an, dass sie für bestimmte soziale Gruppen im selben Kulturkreis einheitlich sind. Er weist außerdem darauf hin, dass Stereotypen eine gewisse ideologische Funktion innewohnt:

They work as justifications for acceptance or rejection of social groups, as well as a device for maintaining simplicity in perception and thinking about groups (Allport 1954), they serve to justify outgroup prejudice and derogation which may lead to ingroup positive distinctiveness (Tajfel 1981), and they serve ideological functions in that they validate the privileges of certain groups over others and justify exploitation because the success and privilege of certain groups are seen as legitimate and 'natural' (Jost and Banaji 1994). Another characteristic feature of stereotypes is that they tend to be shared by group members and are usually identifiable by all members of a particular culture.

(Ladegaard 2011: 87)

Es kann somit zusammenfassend festgehalten werden, dass Stereotype als (mehr oder weniger) sozial verbindliche, Wahrnehmungs- und Evaluationsschemata verstanden werden, über die es in einer bestimmten Gruppe einen Konsens gibt.

Demgegenüber stehen die (sprachlichen) Attitüden. Auch sie werden - je nach Fokus - sehr unterschiedlich gefasst (cf. Garrett 2010: 19). Allport (1954, zit. nach Garrett 2010: 19) beispielsweise beschreibt attitudes als ,a learned disposition to think, feel and behave toward a person (or object) in a particular way“. Im Unterschied zu Stereotypen scheinen Attitüden aber viel mehr von Interaktion geprägt zu sein:

In the reception and production of language too, then, attitudes can be understood in terms of input and output, as a cycle of influence between social cognition and language variation.

Garrett (2010: 22)

Diesen dynamischen, prozessualen Charakter schreibt auch Lenz (2010) Attitüden zu. So hält sie fest: 
They are not innate, predefined and invariable constants, but dynamic and processual features which emerge, further develop and vary in socially interactive processes from sedimentations of individual and transferred experience.

(Deprez/Persoons 1897, zit. nach Lenz 2010: 297; Hervorhebung MF)

Als grundlegend nimmt Lenz für die Attitüden an, dass es sich um Überzeugungen und Wertungen (beliefs and valuations [Lenz 2010: 297]) handelt, was nun noch Hand in Hand mit Stereotypen gehen würde, allerdings sind Attitüden - wie auch aus obigem Zitat hervorgeht individuell geprägt. Die Grenzen zwischen Stereotypen und Attitüden sind damit nicht immer einfach und klar zu ziehen, jedoch sind Stereotype vor allem durch die soziale Verbindlichkeit geprägt - und somit kollektiv angelegt -, Attitüden hingegen können als individuelle Einstellungen, die sich aus (individuellen) Erfahrungen ableiten und verändern, verstanden werden.

Gemeinsam ist Stereotypen und Attitüden aber, dass Bewertungen oder Evaluationen eine große Rolle spielen, da über Bewertungen positive oder negative Einstellungen zum Ausdruck gebracht werden können, die wiederum mit den ,festen Vorstellungen“ (Allport 1971: 200) korrelieren.

Grundsätzlich kann die Bewertung als Opposition zur Beschreibung klassifiziert werden (cf. von Stutterheim/Kohlmann 2001). Weiters kann die Bewertung als Meinungsausdruck beschrieben werden (cf. Bednarek 2006: 187). In diesem Zusammenhang unterscheidet Bednarek (2006: 188) allerdings drei verschiedene Arten von Bewertung:

Consequently, we must make a basic distinction between three notions or definitions of evaluation: (a) the cognitive operation of evaluation, (b), the relatively stable evaluation attached to mental representations, and (c) evaluation as the linguistic expression of speaker/writer opinion.

(Bednarek 2006: 188)

Für die weitere Auseinandersetzung und Analyse in diesem Artikel sind Bewertungen der Gruppe (c) von Bedeutung, wobei von einer Bewertung dann gesprochen werden kann, wenn Attitüden (respektive Stereotype), Haltungen, Ansichten oder Emotionen von SprecherInnen respektive SchreiberInnen zum Ausdruck gebracht werden (cf. Thompson/Hunston 2000: 5, zit. nach Bednarek 2006: 188). Diese Bewertungen können in zehn unterschiedliche bewertende Parameter (evaluative parameters [Bednarek 2006: 188]) eingeteilt werden, die sich auf unterschiedliche Dimensionen beziehen. Daraus ergibt sich folgende Kategorisierung: Verständlichkeit (comprehensibility), Emotivität (emotivity), Wichtigkeit (importance), Ernsthaftigkeit (seriousness), Erwartung (expectedness), mentaler Zustand (mental state), Evidenz (evidentiality), Wahrscheinlichkeit/Notwendigkeit (possibility/necessity), Zuverlässigkeit (reliability) und Stil (style) (cf. Bednarek 2006: 188).

Festzuhalten ist an dieser Stelle, dass Bewertungen, die im Wesentlichen mit Stereotypen und Attitüden Hand in Hand gehen (können), selbst auf komplexe Reflexions- und Bewertungsprozesse zurückzuführen sind:

Tannen suggests that such linguistic expressions are created by expectations or frames (Tannen 1993: 53) (and hence work as indicators of frames), but she also notes that employing evaluative adjectives and adverbs results from and reflects an evaluative process (Tannen 1993: 48). In other words, such expressions are regarded as being both the result of a cognitive operation of evaluation, and as indicators of the existence of mental representations.

(Bednarek 2006: 199; Hervorhebungen im Orig.)

ISSN 1615-3014 


\section{Datenmaterial}

Das Datenmaterial, das für die Analyse herangezogen wird, stammt aus dem Projekt Verknüpfte Analyse von Mehrsprachigkeiten am Beispiel der Universität Salzburg (VAMUS). Dieses wird von Monika Dannerer und Peter Mauser geleitet und setzt thematisch bei der Grundüberlegung an, dass die Universität als international orientierte Bildungseinrichtung als traditionell mehrsprachiger Interaktionsraum gilt. Die gesamtgesellschaftliche Situation ist durch zunehmende Migrationsprozesse, (Bildungs-)Mobilität und Globalisierug geprägt, wodurch Mehrsprachigkeit immer stärker auch als gesellschaftliches „Muss“ angesehen wird, sodass einzelne Menschen gleichzeitig über ganz unterschiedliche Varietäten (,,innere Mehrsprachigkeit") und verschiedene Sprachen (,äußere Mehrsprachigkeit") ${ }^{3}$ (cf. Wandruszka 1979) verfügen. Das Beherrschen unterschiedlicher Sprachen und Varietäten ist dabei unter anderem auf die jeweilige Sozialisation (etwa eine dialektale Prägung durch die Familie oder im Herkunftsort) oder auch auf die Schulbildung (cf. beispielsweise die EU-Forderung, der zufolge EU-BürgerInnen neben der Muttersprache noch zwei weitere EU-Sprachen beherrschen sollen; cf. Europäische Kommission. Generaldirektion Kommunikation 2008: 12) zurückzuführen. Im Alltag dürfte der Umgang mit einer solchen Vielfalt meist unproblematisch sein, die Sprachen scheinen oftmals miteinander in engem Bezug zu stehen. In der linguistischen Forschung jedoch werden Aspekte der inneren und äußeren Mehrsprachigkeit getrennt behandelt. ${ }^{4}$

Im Projekt VAMUS, das eine Laufzeit von vier Jahren hat, werden die jeweiligen Perspektiven auf Mehrsprachigkeit nun aber zusammengeführt. Das Ziel ist es, einen Austausch zwischen bisher getrennten Forschungsrichtungen zu initiieren, letztlich auch (für dieses Projekt) zu vollziehen. Der Ansatz wird am Beispiel der Universität Salzburg ${ }^{5}$ umgesetzt (cf. Dannerer/Mauser 2014, cf. auch Dannerer/Mauser i. Dr.). ${ }^{6}$

Im Forschungsprojekt VAMUS wird vor allem angestrebt, die drei Gruppen - Studierende, wissenschaftliches Personal (Lehrende) und nichtwissenschaftliches Personal - im Hinblick auf ihre

Sprachattitüden, -einschätzungen sowie auf Selbsteinschätzungen sprachlichen Handelns und sprachlicher Entwicklungen zu erfassen und zu analysieren, um ein klares und diastratisch differenziertes Bild von Funktionen und Entwicklungen der Mehrsprachigkeit an der Institution Universität zu gewinnen.

(Dannerer/Mauser 2014)

Neben den Selbsteinschätzungen wird deshalb auch tatsächliches Handeln dokumentiert und mit den Aussagen und Selbsteinschätzungen (vorsichtig) in Bezug gesetzt. Im Sinne der The-

\footnotetext{
${ }^{3}$ Das Konzept der inneren und äußeren Mehrsprachigkeit wurde in Abschnitt 2.1 bereits näher erläutert.

${ }^{4}$ Unterschiedliche Teildisziplinen wie Soziolinguistik, Mehrsprachigkeitsforschung oder Dialektologie setzen jeweils einen anderen Fokus und/oder klammern einen der beiden Bereiche völlig aus.

5 „Die Universität Salzburg ist eine international orientierte Bildungseinrichtung, in der äußere Mehrsprachigkeit eine zentrale Rolle spielt. Gleichzeitig ist die Universität aber auch regional fest verankert: DialektsprecherInnen aus dem unmittelbaren Einzugsgebiet ebenso wie Personen aus dem gesamten deutschen Sprachraum (hier kommen die Formen der inneren Mehrsprachigkeit zum Tragen) und international mobile Menschen mit anderen Erstsprachen als Deutsch aufeinandertreffen, und zwar als Studierende, Forschende und in der Verwaltung“ (Dannerer/Mauser 2014).

${ }^{6}$ Näheres zum Projekt kann auch auf der Projektwebsite unter www.uibk.ac.at/germanistik/vamus/projekt.html [17.03.2016] nachgelesen werden.
} 
ory of Reasoned Action (cf. Garrett 2010: 26-29) ist in diesem Zusammenhang zu berücksichtigen, dass tatsächlich beobachtbares Handeln durch viele unterschiedliche Faktoren beeinflusst sein kann und damit nicht mehr zwingend mit der eigenen Einstellung übereinstimmt. Es ist also nicht unbedingt möglich, eine eindeutige Beziehung zwischen Handlung A und Aussage/Selbsteinschätzung A herzustellen. Die Befunde müssen deshalb jeweils genau geprüft und kritisch hinterfragt werden.

Um das Forschungsvorhaben umsetzen zu können, wird im Rahmen des Projekts ein umfangreiches Datenkorpus erhoben, das sich aus unterschiedlichen Datentypen zusammensetzt. Als Ausgangsbasis wurde schriftliches Datenmaterial zur universitären Sprachenpolitik erhoben, das mit 19 Interviews mit Rektoraten und universitären EntscheidungsträgerInnen ergänzt wurde. Diese Interviews wurden auch an den Universitäten Bozen, Innsbruck und Wien durchgeführt. Die Ausweitung von Salzburg auf Wien und Innsbruck dient dazu, einen tieferen Einblick in den Umgang mit Mehrsprachigkeit an österreichischen Universitäten zu bekommen. Die Universität Bozen wurde aufgrund ihrer explizit verankerten Mehrsprachigkeit ${ }^{7}$ miteinbezogen, um auch die Sichtweisen einer mehrsprachigen Institution einzufangen.

Grundsätzlich wird im Projekt Triangulation auf allen Ebenen (sowohl bezüglich der Daten als auch der Methoden) angestrebt. So werden quantitative Methoden eingesetzt, um unter anderem grundlegende Attitüden zum Thema Mehrsprachigkeit, wie sie zuvor in Kapitel 2.1 definiert wurde, mittels Online-Fragebögen zu erheben. Der Pool an Fragebögen umfasst ca. 1250 Datensätze, die sich auf die drei oben genannten Gruppen aufteilen. Zusätzlich dazu wurden ca. 120 Tiefeninterviews in den drei Untersuchungsgruppen (Studierende, Lehrende, nicht-wissenschaftliches Personal) durchgeführt, die qualitative Einblicke geben, aber auch mit den Erkenntnissen der quantitativen Erhebung verknüpft werden können.

In einem letzten Erhebungsschritt wurden ca. 25 Video- beziehungsweise Audioaufnahmen in Lehrveranstaltungen sowie bei Gesprächen in und mit der Verwaltung gemacht, die tatsächliches sprachliches Handeln dokumentieren. Sie werden diskursanalytisch ausgewertet und mit den Ergebnissen der anderen Erhebungsstufen in Bezug gesetzt.

Für den vorliegenden Beitrag wird das VAMUS-Teilkorpus „Interviews mit Rektoraten und EntscheidungsträgerInnen“, das von Sebastian Maier ${ }^{8}$ erhoben wurde, für die Analyse herangezogen. Es werden erste Teilergebnisse präsentiert, wobei der inhaltliche Fokus auf kollektiven und individuellen Vorstellungen zu Mehrsprachigkeiten liegt.

\section{$4 \quad$ Fragestellungen und Methoden}

Wenn nun in Abschnitt 2 auf einer theoretischen Basis Stereotyp und Attitüde definiert und voneinander abgegrenzt wurden, so ist noch offen, ob diese relativ klare Differenzierung auch in der Praxis haltbar ist.

\footnotetext{
${ }^{7}$ An der Universität Bozen sind die drei Sprachen Deutsch, Italienisch und Englisch offizielle Institutionssprachen.

${ }^{8}$ Sebastian Maier hat mir für diese Arbeit dankenswerterweise die von ihm geführten Interviews zur Verfügung gestellt. Weitere Auswertungen der hier behandelten Daten wird Maier in den nächsten Monaten in seiner Masterarbeit (i. V.) zugänglich machen.
} 
Weiters ist zu fragen:

- Treten Stereotyp und Attitüde getrennt voneinander auf oder sind sie auch in gleichen Gesprächsabschnitten zu finden?

- Welche Funktionen kommen dem Stereotyp, welche der Attitüde zu?

Diesen Fragen liegt die hypothetische Annahme zugrunde, dass Stereotype vor allem dann eingesetzt werden, wenn der Meinung einer Community Ausdruck verliehen wird. Attitüden hingen werden zur Untermauerung der eigenen Sichtweise eingesetzt. Es wird zudem davon ausgegangen, dass vor allem Stereotype funktionalisiert werden, um sich als Teil einer Gemeinschaft zu präsentieren.

Um diese Fragen und Hypothesen beantworten zu können, wird ein inhaltsanalytischpragmatischer Ansatz gewählt. Zur Analyse wurden exemplarisch Beispiele aus dem VAMUS-Teilkorpus „Interviews mit Rektoraten und EntscheidungsträgerInnen“ (als Leitfadeninterviews von Sebastian Maier geführt) herangezogen, die nach GAT 2 (cf. Selting et al. 2009) transkribiert wurden.

Thematisch wurden sowohl Beispiele aus dem Bereich der äußeren, als auch aus dem Themengebiet der inneren Mehrsprachigkeit untersucht. Inhaltlich steht in allen ausgewählten Beispielen der universitäre Diskurs im Fokus. Im Kontext der Internationalisierung sind die Themenbereiche „Englisch als Wissenschaftssprache“ sowie „Varietäten des Deutschen und deren Verständlichkeit“" immer wieder zentral.

\section{$5 \quad$ Analyse}

Die exemplarische Analyse erfolgt zunächst nach Themenbereichen getrennt, wobei im Themenbereich der äußeren Mehrsprachigkeit zwei Gesprächsausschnitte ausgewählt wurden, in denen es um die Rolle des Englischen in der Wissenschaft bzw. an der Universität geht. Ein drittes Beispiel fokussiert dann inhaltlich auf Aspekte der inneren Mehrsprachigkeit.

\subsection{Englisch als vernetzende Sprache}

\section{Beispiel $1^{9}$}

VAMUS-Teilkorpus „Interviews mit Rektoraten und EntscheidungsträgerInnen“

Interview: SM, 30.06.2014

Interviewpartner B2: Freie Universität Bozen, obere Managementebene

Gesamtlänge des Interviews: 00:13:10, Ausschnitt: 00:03:24 - 00:04:15

Transkription: MF.

Im folgenden Interviewausschnitt wird die Rolle des Englischen in der Wissenschaft thematisiert. Diese Frage wird eher zu Beginn des Gespräches (ca. 00:03:24 - 00:04:15) erläutert. Zuvor wurde die mehrsprachige Situation an der Freien Universität Bozen besprochen sowie

\footnotetext{
${ }^{9}$ Die Sprechersiglen setzen sich aus dem Kürzel für den Universitätsstandort sowie einer Zahl, die die Funktion verschlüsselt, zusammen. Diese Zahl drückt aber keinesfalls die tatsächliche Hierarchie aus, sondern wurde willkürlich vergeben.
} 
die Forderung der EU-Kommission nach Mehrsprachigkeit (cf. Abschnitt 3.1) diskutiert. Im Anschluss an den hier gezeigten Ausschnitt wird thematisiert, inwieweit Englisch eine (sprachliche) Hürde sein könnte und ob eine etwaige muttersprachliche Kompetenz Vorteile/Nachteile bringen kann.

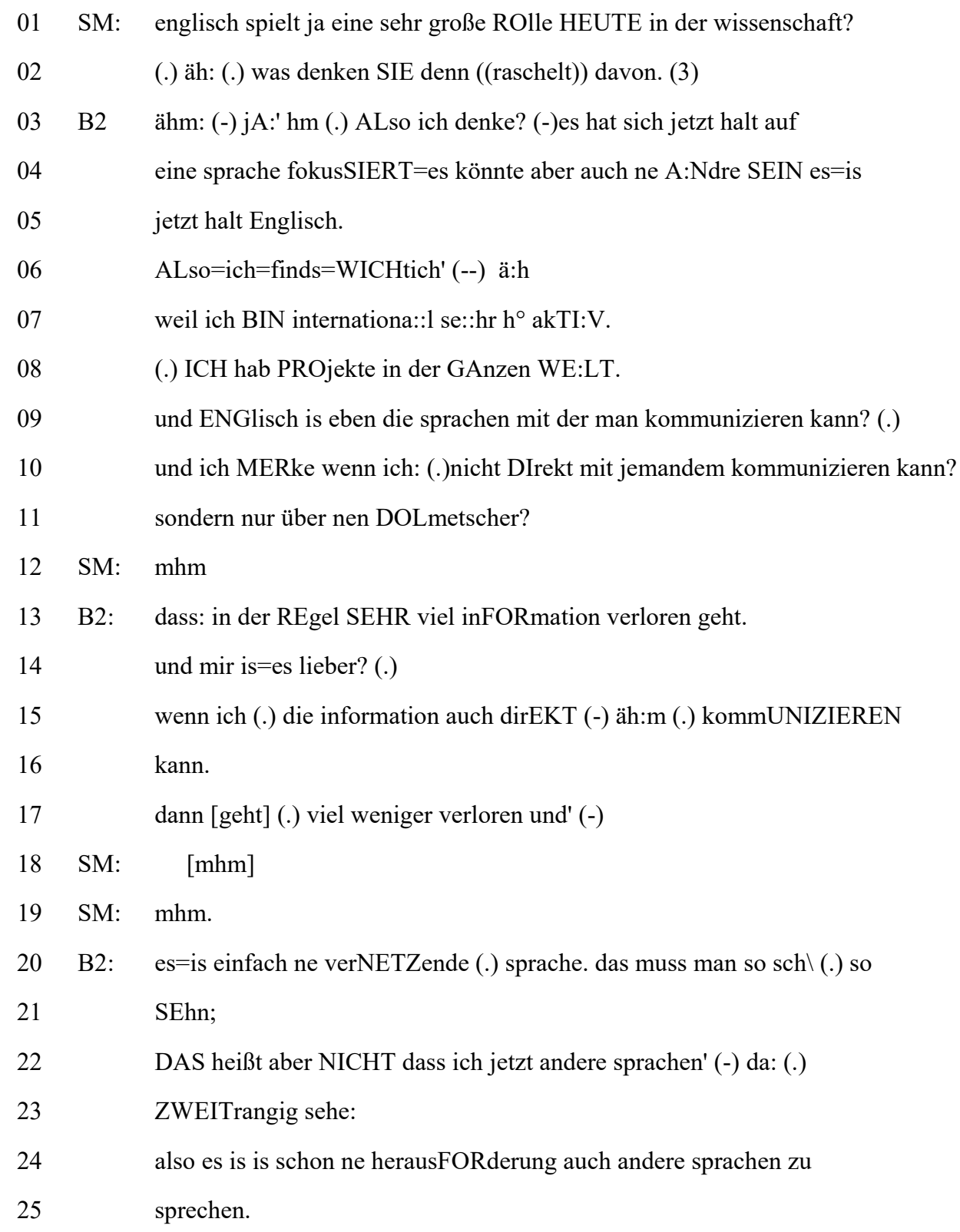

In diesem Abschnitt wird über den Einsatz des Englischen und die Auswirkungen davon gesprochen, wobei der Status von Englisch in Zeile 6 zunächst evaluiert wird. Anschließend gibt es zu dieser Bewertung vom Sprecher noch nähere Erläuterungen bzw. Begründungen (Zeile 7-17).

Dabei können sowohl Attitüden als auch Stereotype festgehalten werden: Die Evaluierung in Zeile 6 beispielsweise, die nach Bednarek (2006: 192) dem Parameter des mentalen Zustands (Erwartung) entspricht, ist den Attitüden zuzuordnen, da hier in der Ich-Perspektive eine individuelle Meinung präsentiert wird (,,ich find's wichtig“").

Wie schon in Abschnitt 2.2 ausgeführt wurde, hängen Stereotyp, Attitüde und Bewertung eng zusammen. Hier zeigt sich sogar, dass sie sich mitunter bedingen: Mit der Modalpartikel 
„eben“ in Zeile 9 kann dies verdeutlicht werden. So ist diese nach Bednarek (2006: 188-189) den drei Parametern der Wichtigkeit (die Bedeutung der Sprache Englisch wird unterstrichen), der Wahrscheinlichkeit/Notwendigkeit sowie des mentalen Zustands (Erwartung) zuzuordnen. Gleichzeitig liegt für diese Proposition eine prädikative Konstruktion vor (,und Englisch ist"), wodurch die Bewertung, die auch subjektiven, individuellen Charakter haben könnte, den Status der Allgemeingültigkeit - und damit eines Stereotyps (im Sinne eines reduzierten Evaluationsschemas) - bekommt.

Ähnliches gilt auch für die Modalpartikel „einfach“ in Zeile 20. Die Aussage „Es ist einfach eine vernetzende Sprache.", mit der diese begründende Passage endet, ist zusätzlich noch mit der Proposition „Das muss man so sehen.“ gekoppelt, die durch die Verbindung der beiden Sätze zum Ausdruck eines Stereotyps wird. Durch den bewertenden Charakter kann der Satz „Das muss man so sehen.“ nicht mehr als objektive Darstellung einer Tatsache gewertet werden. Außerdem wird die Aussage „Es ist einfach eine vernetzende Sprache.“ nicht mehr ausgehandelt/begründet, sondern als ,gegeben“ angesehen, weshalb sie ebenfalls als Stereotyp zu klassifizieren ist. Es wird vom Sprecher somit angenommen, dass darüber ein Konsens vorliegt. Diese Proposition entspricht also einem reduzierten Evaluationsschema. Von einer reinen Tatsache/Behauptung hebt sich die Aussage durch das adjektivische Attribut „,vernetzen$\mathrm{de}^{\text {" }} \mathrm{ab}$.

In Zeile 22 beginnt der Sprecher dann, seine persönliche Einstellung zum Thema zu referieren, was der Äußerung einer Attitüde entspricht. Er endet schließlich erneut mit einer Verallgemeinerung: „Es ist schon einen Herausforderung, auch andere Sprachen zu sprechen.“ Diese Proposition entspricht wiederum einem reduzierten Evaluationsschema, es bleibt allerdings etwas unklar, ob inhaltlich auch ein (sozialer) gruppenspezifischer Konsens vorliegt, wobei der Sprecher dies so präsentiert.

Zusammengefasst kann damit für dieses Beispiel konstatiert werden, dass Attitüden und Stereotype gemeinsam auftreten und in einem gewissen Wechselspiel zueinander stehen.

\subsection{Mehrsprachigkeit als Reduktion auf Englisch}

Auch im nächsten Interviewausschnitt kann ein Wechselspiel und Ineinandergreifen von Attitüden und Stereotypen beobachtet werden.

\section{Beispiel 2}

VAMUS-Teilkorpus „Interviews mit Rektoraten und EntscheidungsträgerInnen“

Interview: SM, 12.08.2014

Interviewpartnerin S5: Universität Salzburg, mittlere Managementebene

Gesamtlänge des Interviews: 00:26:30, Ausschnitt: 00:02:04 - 00:02:39

Transkription: MF.

$\mathrm{Zu}$ Beginn des Gespräches geht es darum, inwieweit S5 ihr Arbeitsumfeld als mehrsprachig bezeichnet. In diesem Zusammenhang beginnt die Interviewte, von ihrem Forschungsprojekt zu erzählen, an dem 33 Staaten beteiligt sind. Es wird weiters thematisiert, welche Sprachen neben Deutsch und Englisch noch eine Rolle spielen. Der nachfolgende Ausschnitt steht wiederum am Anfang des Gespräches (ca. 00:02:04 - 00:02:39). Im Anschluss an die hier analy- 
sierte Passage geht es unter anderem um wissenschaftliche Projekte, internationale Publikationsmöglichkeiten (Citation-Index) und die lingua franca Englisch.

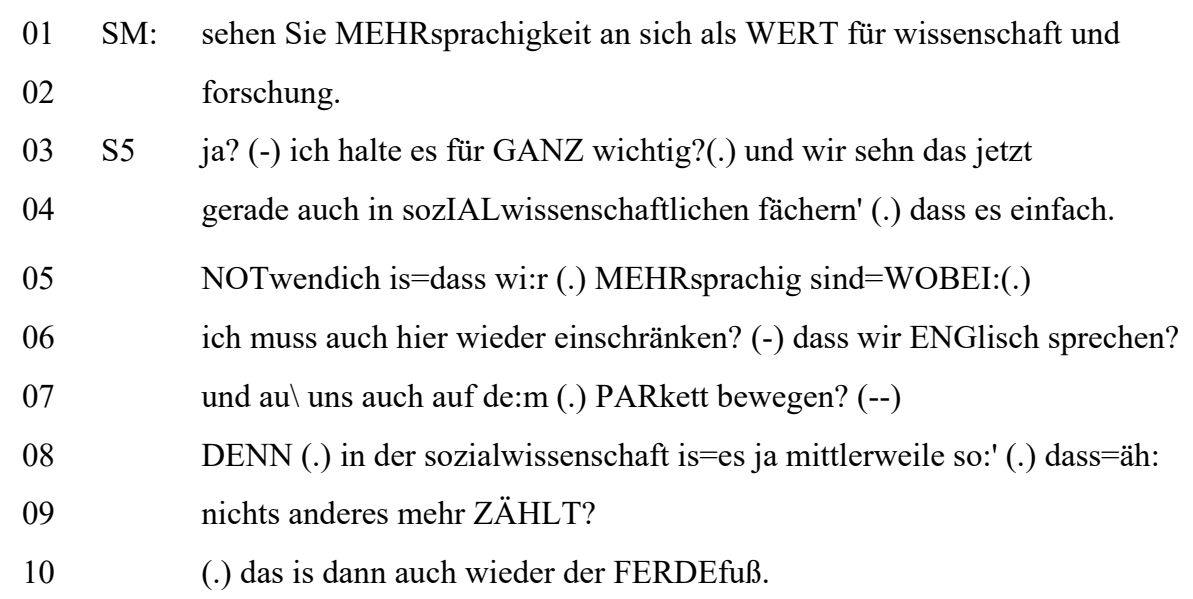

In Zeile 3 kann die Antwort von S5 noch recht klar als Attitüde klassifiziert werden (,,ich halte es für“" zeigt einen individuellen Bezug an). Dies ist die Antwort auf die vom Interviewer gestellte Frage, die klar auf die individuelle Einstellung zu diesem Thema (Mehrsprachigkeit als Wert für die Wissenschaft) - und damit also auf Attitüden - abzielt.

Wie schon zuvor in Beispiel 1 spielen auch in diesem Ausschnitt bewertende Ausdrücke/Elemente eine Rolle. In Zeile 4-5 wird - ähnlich wie auch in Beispiel 1 - die Modalpartikel „einfach“ dazu genutzt, stereotype Vorstellungen zum Ausdruck zu bringen. Hier kann „einfach“ dem Parameter der Wahrscheinlich/Notwendigkeit (cf. Bednarek 2006; siehe auch Abschnitt 2.2) zugeordnet werden. In Zeile 5 erläutert die Sprecherin dann die Notwendigkeit, mehrsprachig zu sein, näher und wird durch die prädikative Konstruktion („dass es einfach notwendig ist") absolut in ihrer Wertung und Bezugnahme, die mit der Modalpartikel eingeleitet wurde.

Auffällig ist zudem der Wechsel vom Personalpronomen der 1. Person Sg. „ich“ am Beginn der dritten Zeile zum Plural ,wir“ am Ende derselben Zeile. Dadurch wird ein Perspektivenwechsel angezeigt, die Sprecherin vertritt also nicht mehr ihre individuelle Meinung, sondern die Meinung einer bestimmten Gruppe, eines Kollektivs. (Die Einschränkung auf Englisch passiert interessanterweise wieder in der Ich-Form). Der Perspektivenwechsel wird zusätzlich durch die Konstruktion mit Korrelat „es“ und Prädikativ „ist“ vollzogen.

Dieser Teil ist somit klar als Stereotyp zu werten, da keine individuellen Erfahrungen präsentiert werden. Dies wird vor allem durch das „wir“ (Zeile 3, 5 und 6) deutlich, wie bereits zuvor argumentiert wurde. Die Aussage wird durch ihre Präsentationsform sozial verbindlich für eine gewisse Gruppe (eben die Sozialwissenschaften bzw. einen bestimmten Teil davon). Man könnte nun natürlich auch davon ausgehen, dass der ganze hier präsentierte Turn als individuelle Meinungskundgabe - und damit als Attitüde - zu werten ist. Dies scheint mir in der Analyse jedoch zu einfach, vielleicht auch etwas zu oberflächlich zu sein, da gerade an dieser Stelle deutlich wird, wie eng Attitüden und Stereotype miteinander verbunden sind und wie vielschichtig ein Turn diesbezüglich sein kann.

Auch die Äußerung in Zeile 9-10 kann als Stereotyp gewertet werden, wobei durch das Temporaladverb „mittlerweile“ zusätzlich eine Entwicklung zum Ausdruck gebracht wird. 
Der Transkriptausschnitt endet dann mit einem Stereotyp: „Das ist dann auch wieder der Pferdefuß“, der sich auf den Einsatz von Englisch als (einzige) lingua franca bezieht. Durch die prädikative Konstruktion wird diese Proposition als reduziertes Evaluationsschema geäußert, gleichzeitig bleibt aber - wie schon im vorherigen Analysebeispiel - offen, ob darüber auch in einer (größeren) Gruppe Konsens herrscht.

\subsection{Vorstellungen zu Varietäten des Deutschen in Österreich}

Das nun folgende Beispiel fokussiert thematisch auf Perspektiven der inneren Mehrsprachigkeit. Auch hier können Aspekte in Bezug auf Stereotyp und Attitüde beobachtet werden, die schon in den beiden vorigen Analyseausschnitten deutlich wurden.

\section{Beispiel 3}

VAMUS-Teilkorpus „Interviews mit Rektoraten und EntscheidungsträgerInnen“

Interview: SM, 14.07.2014

Interviewpartner 19: Universität Innsbruck, obere Managementebene

Gesamtlänge des Interviews: 00:28:36, Ausschnitt: 00:19:43 - 00:22:22

Transkription: Ersttranskription: SM, Überarbeitung und Ergänzung: MF.

Auch in diesem Interview wird zu Beginn thematisiert, inwieweit I9 sein Arbeitsumfeld als mehrsprachig ansieht. Außerdem wird diskutiert, inwiefern Mehrsprachigkeit wichtig für die Wissenschaft und als Potenzial für die Universität zu sehen ist. Auch geforderte Sprachniveaus werden besprochen. Weiters sind die Rolle des Englischen als lingua franca sowie Migrationssprachen an der Universität Thema. Nachfolgender Transkriptausschnitt stammt aus dem letzten Drittel des Gesprächs (ca. 00:19:43 - 00:22:22). Direkt vor dieser Passage geht es um sprachliche Herausforderungen für (ERASMUS)StudentInnen. Im Anschluss an den vorliegenden Ausschnitt werden die „Standardkompetenzen“ der SüdtirolerInnen noch weiter diskutiert.

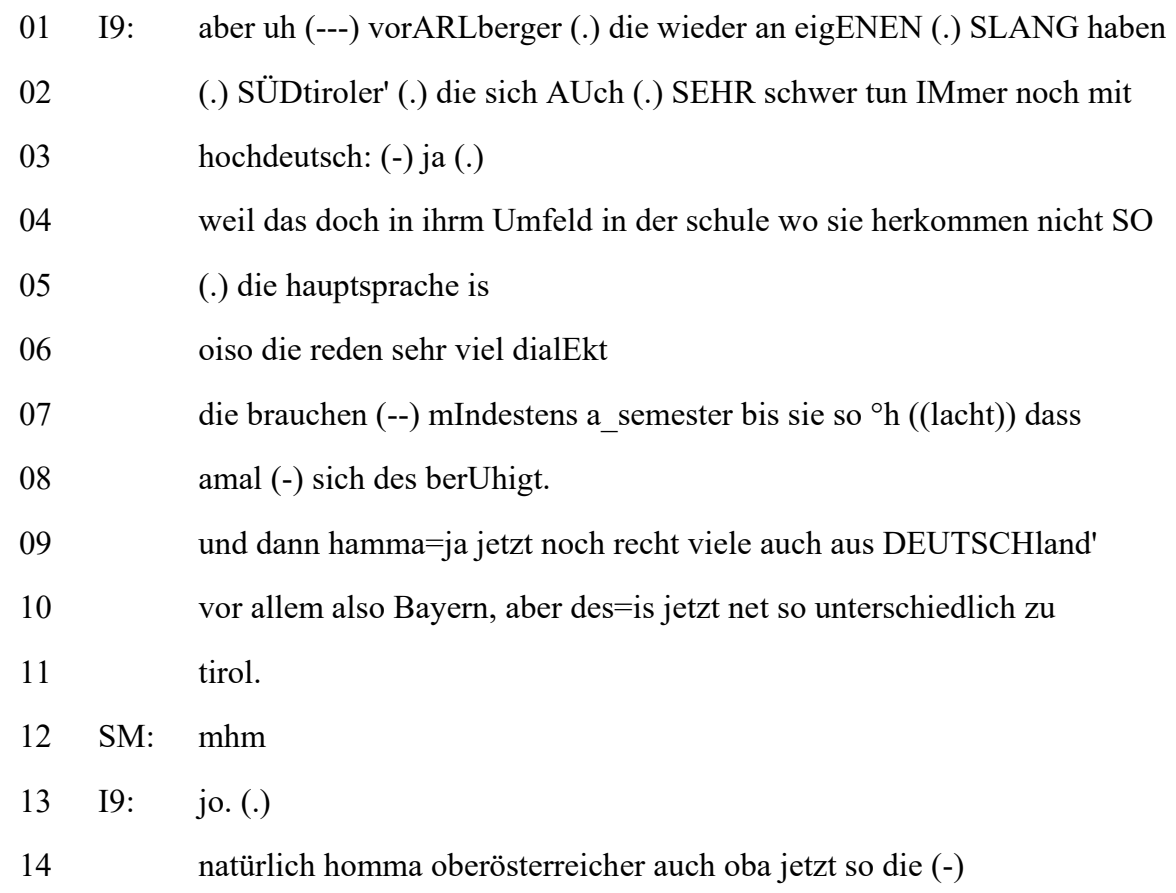



vorARLberg (.) und südtirol ja.

In diesem Transkriptausschnitt ist ebenfalls eine Wechselbeziehung zwischen Attitüden und Stereotypen zu beobachten. Anders als in den vorigen Beispielen beginnt der Sprecher aber mit einem Stereotyp: Zeile 1-3. Die Aussage, die der Sprecher über SprecherInnen des Vorarlberger und Südtiroler „Slangs“ (er meint wahrscheinlich Dialekt, die eigentliche Bedeutung bleibt aber unklar) trifft, kann als reduziertes Wahrnehmungsschema - und damit als Stereotyp - eingestuft werden. Interessant ist, dass I9 für seine Wertung auch eine Begründung angibt, die wiederum als Stereotyp klassifiziert werden kann (Zeile 4-5). So geht er davon aus bzw. präsentiert es als Tatsache, dass für VorarlbergerInnen und SüdtirolerInnen Hochdeutsch in der Schule sowie in ihrem (sozialen) Umfeld keine Rolle spielt.

Auch die Klassifizierung der Bayern, die in Bezug auf das Sprechen nicht so anders seien als Tiroler, ist als Stereotyp zu klassifizieren, was auf sprachlicher Ebene in der prädikativen Konstruktion deutlich wird.

Am Ende scheint der Sprecher seine Aussagen wieder etwas zu relativieren. Durch die geäuBerte Attitüde in Zeile 15-16, die mit „,ich glaube“ eingeleitet ist, wird es zudem fraglich, ob zum Rest der Passage ein allgemeiner Konsens vorherrscht, oder ob der Sprecher hier seine persönliche Meinung im Licht der Allgemeingültigkeit präsentiert.

\section{$6 \quad$ Fazit}

Wie sich in den Beispielen gezeigt hat, treten Attitüden und Stereotype oftmals gemeinsam auf, womit die zu Beginn gestellte Frage nach dem gemeinsamen Vorkommen von Stereotyp und Attitüden klar beantwortetet werden kann. Auffällig ist dabei, dass sie häufig sehr eng miteinander verbunden sind, was in dieser Analyse mit „Wechselspiel“ angedeutet wurde.

Gleichzeitig hat sich aber auch gezeigt, dass die auf der theoretischen Basis relativ klare Abgrenzung zwischen Stereotyp und Attitüde in der sprachlichen Praxis meist relativ gut möglich ist, dass aber beispielsweise durch die Verwendung prädikativer Konstruktionen individuelle Einstellungen - und damit Attitüden - auch im Licht der Allgemeingültigkeit und eines gesellschaftlichen Konsens - und damit als Stereotyp - präsentiert werden können.

Dies steht in engem Zusammenhang mit unterschiedlichen Funktionen von Stereotypen und Attitüden im Diskurs, die deutlich wurden: So werden Stereotype tendenziell wohl dazu genutzt, der Aussage mehr Gewicht zu geben und die individuelle Einstellung/Attitüde noch zu untermauern. Umgekehrt kann die Attitüde dazu genutzt werden, das zuvor Gesagte (als Stereotyp Geäußerte) etwas abzuschwächen und in Richtung individuelle Meinung/Einstellung $\mathrm{zu}$ verschieben. Interessanterweise treten (subtile) Bewertungen (etwa in Form von Modalpartikeln) in den hier analysierten Transkriptausschnitten nur im Kontext von Stereotypen auf.

Die zu Beginn gestellte Hypothese, dass Stereotype vor allem dann eingesetzt werden, wenn der Meinung einer Community Ausdruck verliehen wird, wohingegen Attitüden zur Untermauerung individueller Perspektiven eingesetzt werden, kann damit verifiziert werden. Auch die Hypothese, dass Stereotype und Attitüden funktionalisiert werden, wurde bestätigt. 
Nach der Durchsicht des hier herangezogenen Teilkorpus kann der vorliegende Befund als typisch eingestuft werden. Somit haben die drei Ausschnitte für diese Korpus durchaus repräsentativen Charakter. Es gilt dies allerdings mit einer umfangreicheren Analyse noch weiter zu überprüfen. Eine breitere Untersuchung in diese Richtung scheint durchaus sinnvoll und gewinnbringend zu sein. Ein Desiderat ist in diesem Zusammenhang auch, die Abgrenzung zwischen Attitüde und Stereotyp noch deutlicher zu machen und mit umfangreicheren Analysen zu belegen.

\section{Literatur}

Allport, Gordon Williard (1954): "The historical background of modern social psychology". In: Lindzey, Gardner (ed.): Handbook of social psychology. Bd. 1. Theory and method. Cambridge, Addison-Wesley: 3-56.

Allport, Gordon Williard (1971): Die Natur des Vorurteils. Hrsg. und komm. von Carl Friedrich Graumann. Köln: Kiepenheuer \& Witsch.

Auer, Peter (2007): “Introduction”. In: Auer, Peter (ed.): Style and Social Identities. Alternative Approaches to Linguistic Heterogeneity. Berlin/New York, Mouton de Gruyter: 1-21.

Bednarek, Monika (2006): "Evaluation and cognition: Inscribing, evoking and provoking opinion". In: Pishwa, Hanna (ed.): Language and Memory. Aspects of Knowledge Representation. Berlin/New York, de Gruyter: 187-222.

Bourdieu, Pierre (2012): Was heißt sprechen? Zur Ökonomie des sprachlichen Tausches. Mit einer Einführung von John B. Thompson. Übersetzt aus dem Französischen von Hella Beister. Wien: New Academic Press.

Busch, Brigitta (2012): “The Linguistic Repertoire Revisited”. Applied Linguistics 33/5: 503523.

Busch, Brigitta (2013): Mehrsprachigkeit. Wien: Facultas.

Dannerer, Monika (2008): „Die Wissenschaft spricht Englisch. Und Deutsch?!“ In: Nicolini, Maria (ed.): Wissenschaft, helldunkler Ort. Sprache im Dienst des Verstehens. Wien, Braumüller: 59-68.

Dannerer, Monika (2015): „Gewünschte, gelebte und verdeckte Mehrsprachigkeit an der Universität“. ÖDaF-Mitteilungen 2 (Themenheft: Dem Lernen auf der Spur): 143-150.

Dannerer, Monika/Mauser, Peter (2013): Verknüpfte Analyse von Mehrsprachigkeiten am Beispiel der Universität Salzburg (VAMUS). Unveröffentlichter Projektantrag.

Dannerer Monika/Mauser, Peter (2014): Verknüpfte Analyse von Mehrsprachigkeiten am Beispiel der Universität Salzburg (VAMUS). www.uibk.ac.at/germanistik/vamus/projekt beschreibung [01.10.2015].

Dannerer Monika/Mauser, Peter (2016): „Österreichische Universitäten als mehrsprachige Interaktionsräume? Universitäre Sprachenpolitik vor dem Hintergrund des Projektes ,Verknüpfte Analyse von Mehrsprachigkeiten am Beispiel der Universität Salzburg (VAMUS)““. In: Jablkowska, Joanna/Kupczynska, Kalina/Müller, Stephan (eds.) (2016): Literatur, Sprache und Institution. Wien: Präsens.

Deprez, Kas/Persoons; Yves (1987): „Attitude“. In: Ammon, Ulrich/Dittmar, Norbert/Mattheier, Klaus J. (eds.): Soziolinguistik. Ein internationales Handbuch zur Wissenschaft von Sprache und Gesellschaft. Bd. 1. Berlin, de Gruyter: 125-132. 
(= Handbücher zur Sprach- und Kommunikationswissenschaft / Handbooks of Linguistics and Communication Science (HSK))

Europäische Kommission. Generaldirektion Kommunikation (ed.) (2008): Viele Sprachen für ein Europa. Sprachen in der Europäischen Union. Luxemburg: Amt für amtliche Veröffentlichungen der Europäischen Gemeinschaft.

Franceschini, Rita (2011): "Multilingualism and Multicompetence: A Conceptual View". The Modern Language Journal 95/3: 344-355.

Garrett, Peter (2010): Attitudes to Language. Cambridge: Cambridge University Press.

Jost, John/Banaji, Mahzarin (1994): "The role of stereotyping in system-justification and the production of false consciousness”. British Journal of Social Psychology 33: 1-27.

Kaufmann, Götz (1997): Varietätendynamik in Sprachkontaktsituationen: Attitüden und Sprachverhalten rußlanddeutscher Mennoniten in Mexiko und den USA. Frankfurt a. M.: Lang.

Klein, Josef (1998): „Linguistische Stereotypbegriffe. Sozialpsychologischer vs. semantiktheoretischer Traditionsstrang und einige frametheoretische Überlegungen“. In: Heinemann, Margot (ed.): Sprachliche und soziale Stereotype. Frankfurt a. M., Lang: 25-46.

Konerding, Klaus-Peter (2001): „Sprache im Alltag und kognitive Linguistik: Stereotype und schematisiertes Wissen“. In: Lehr, Andrea et al. (eds.): Sprache im Alltag. Beiträge zu neuen Perspektiven in der Linguistik. Herbert Ernst Wiegand zum 65. Geburtstag gewidmet. Berlin/New York, de Gruyter: 151-185.

Ladegaard, Hans J. (2011): "Stereotypes And The Discursive Accomplishment Of Intergroup Differentiation: Talking About 'The Other' In A Global Business Organization”. Pragmatics 21/1: 85-109.

Lenz, Alexandra N. (2010): "Emergence of Varieties through Restructuring and Reevaluation”. In: Auer, Peter/Schmidt, Jürgen Erich (eds.): Language and Space. An International Handbook of Linguistic Variation. Volume 1: Theories and Methods. Berlin/New York, Mouton de Gruyter: 295-315. (= Handbücher zur Sprach- und Kommunikationswissenschaft / Handbooks of Linguistics and Communication Science (HSK)).

Maier, Sebastian (i. V.): Sprachenpolitik an ausgewählten Hochschulen in Österreich und Südtirol (Arbeitstitel). Nicht publizierte Masterarbeit. Universität Salzburg.

Pümpel-Mader, Maria (2010): Personenstereotype. Eine linguistische Untersuchung zu Form und Funktion von Stereotypen. Heidelberg: Winter.

Putnam, Hilary (1975): “The Meaning of 'Meaning'”. In: Gunderson, Keith (ed.): Language, mind and knowledge. Minneapolis, University of Minnesota Press: 131-193.

Quasthoff, Uta (1973): Soziales Vorurteil und Kommunikation - Eine sprachwissenschaftiche Analyse des Stereotyps. Ein interdisziplinärer Versuch im Bereich von Linguistik, Sozialwissenschaft und Psychologie. Frankfurt/M.: Fischer.

Quasthoff, Uta (1978): “The Uses of Stereotypes in everyday Argument”. Journal of Pragmatics 2: 1-48.

Quasthoff, Uta (1998): „Stereotype in Alltagssituationen: Ein Beitrag zur Dynamisierung der Stereotypenforschung“. In: Heinemann, Margot (ed.): Sprachliche und soziale Stereotype. Frankfurt a. M., Lang: 47-72.

Schiewe, Jürgen (1996): Sprachenwechsel - Funktionswandel - Austausch der Denkstile. Die Universität Freiburg zwischen Latein und Deutsch. Tübingen: Niemeyer. 
Selting, Margret et al. (2009): „Gesprächsanalytisches Transkriptionssystem 2 (GAT 2)“. Gesprächsforschung - Online-Zeitschrift zur verbalen Interaktion 10: 353-402.

Stutterheim, Christiane von/Kohlmann, Ute (2001): „Beschreiben im Gespräch“. In: Brinker, Klaus/Antos, Gerd/Heinemann, Wolfgang (eds.): Text- und Gesprächslinguistik. Linguistics of Text and Conversation. Ein internationales Handbuch zeitgenössischer Forschung. An international Handbook of Contemporary Research. 2. Halbband. Berlin/New York, de Gruyter: 1279-1292.

Tajfel, Henri (1981): "Social stereotypes and groups". In: Turner, John/Giles, Howard (eds.): Intergroup Behavior. Oxford, Blackwell: 132-145.

Tannen, Deborah (1993): "What's in a frame?: Surface evidence for underlying expectations". In: Tannen, Deborah (ed.): Framing in Discourse. Oxford, Oxford University Press: $14-57$.

Thomson, Geoff/Hunston, Susan (2000): "Evaluation. An introduction". In: Hunston, Susan/Thomson, Geoff (eds.): Evaluation in Text: Authorial Stance and the Construction of Discourse. Oxford, Oxford University Press: 1-27.

Wandruszka, Mario (1979): Die Mehrsprachigkeit des Menschen. München: Piper. 